no points for improvement concerning the tools. Patients commented on the website, and changes were made accordingly. Discussion We developed a tailor-made strategy for PPH guideline implementation. The next step in the implementation process is to evaluate the feasibility of the strategy, including an effect, process and cost evaluation.

\section{P247 A SIMPLE GUIDELINE APPRAISAL INSTRUMENT BASED ON IOM STANDARDS}

${ }^{1,2} \mathrm{M}$ Mitchell, ${ }^{1,2} \mathrm{~B}$ Leas, ${ }^{1,2}$ J Lavenberg, ${ }^{1,4,8} \mathrm{D}$ Goldmann, ${ }^{1,2,4,5,6,7} \mathrm{C}$ Umscheid. ${ }^{1} \mathrm{C}$ enter for Evidence-based Practice, University of Pennsylvania Health System, Philadelphia, USA; ${ }^{2} E C R I$ Institute-Penn Medicine Evidence-based Practice Center, Philadelphia, USA; ${ }^{3}$ Clinical Practices of the University of Pennsylvania, Philadelphia, USA; ${ }^{4}$ Department of Medicine, University of Pennsylvania School of Medicine, Philadelphia, USA; ${ }^{5}$ Leonard Davis Institute of Health Economics, Philadelphia, USA; ${ }^{6}$ Center for Clinical Epidemiology and Biostatistics Philadelphia, USA; 7 Institute for Translational Medicine and Therapeutics Philadelphia USA Elsevier Health Sciences Philadelphia, USA

\section{0:1136/bmjqs-2013-002293.226}

Background Scales like AGREE provide a systematic means for appraising guideline quality, but they are lengthy, emphasise methodology over practicality, and are best applied by guideline experts.

Objectives Create a short instrument for guideline appraisal, based on widely accepted standards.

Methods The Institute of Medicine (IOM) identified eight principles that make a guideline 'trustworthy'. We adapted each principle into an item graded 'A', 'B', 'C', or "NR" (not reported). Guideline assessments are presented as a grid rather than a single score, with each row representing an item, each column a guideline, and cells coloured green, yellow, red or white to reflect the above grades, respectively. Concordance tables mapping AGREE and G-I-N standards to IOM domains were also created.

Results Piloted use of the tool suggests it can distinguish guidelines developed using weak methods and those that are poorly documented. Grids highlight guideline strengths and weaknesses, as well as guidelines that are more trustworthy than their comparators. The concordance table found that AGREE lacks standards for guideline currency and updating, while IOM lacks standards for resource implications.

Discussion Our pilot use of this instrument suggests that while the overall trustworthiness of guidelines is important, using IOM domains to understand sources of guidelines' weaknesses can help organisations select guidelines best suited for their needs. Further work will examine our instrument's reliability across users with different levels of expertise.

Implications for Guideline Developers/Users Pilot use of this tool suggests it can be applied by clinicians and administrators who have limited training and time.

\section{P260 A MODEL FOR BRIDGING THE TRANSLATIONAL VALLEY OF DEATH IN SPINAL CORD INJURY}

${ }^{1} \mathrm{~B}$ Barrable, ${ }^{1} \mathrm{~N}$ Thorogood, ${ }^{1,2} \mathrm{~V}$ Noonan, ${ }^{1} \mathrm{P}$ Joshi, ${ }^{1} \mathrm{~K}$ Stephenson, ${ }^{2} \mathrm{~B}$ Kwon, ${ }^{1,2} \mathrm{M}$ Dvorak. ${ }^{1}$ Rick Hansen Institute, Vancouver, Canada; 2 Division of Spine, Department of Orthopedics, University of British Columbia, Vancouver, Canada

\section{0:1136/bmjqs-2013-002293.227}

Background Despite the amount of funding that supports basic research, few research discoveries achieve their potential. The transition from bench-to-bedside research is so fraught with obstacles that it is referred to as the "valley of death".

Objective The Rick Hansen Institute (RHI) developed a unique Praxis Model for translational research in the field of spinal cord injury (SCI). At RHI this means bringing knowledge into action; to improve healthcare outcomes for people with SCI and decrease the financial impact on the healthcare system.

Methods The research continuum begins with discovery science which feeds into the knowledge cycle, continues with the acceptance and uptake into the treatment of spinal cord injuries. The core activity within the Praxis Model is a knowledge cycle that consists of a four-phased strategy: 1) Environmental scan, 2) Knowledge generation and synthesis, 3) Knowledge validation, and 4) Implementation.

Results RHI has participated and supported over 60 studies since 2007 and engaged researchers from nine countries, 46 academic institutions and various accreditation and professional associations. Currently, the model is being independently evaluated to determine strengths and limitations. Examples of RHI initiatives using the Praxis Model and results of the evaluation will be presented.

Discussion RHI has developed an innovative solution to move knowledge into action. The Praxis Model strives to lead collaboration across the global SCI community by providing funding, infrastructure, strategic partnerships, governance and a network.

Implications Lessons learned in developing the Praxis Model may assist other organisations dealing with similar translational research challenges.

\section{P263 IMPLEMENTING A NEW STANDARD FOR MEDICAL SPECIALTY SOCIETY GUIDELINE DEVELOPMENT}

${ }^{1} \mathrm{C}$ Wolfkiel, ${ }^{2} \mathrm{~W}$ Smith Begolka, ${ }^{3} \mathrm{G}$ Fulda. ${ }^{1}$ American College of Occupation and Environmental Medicine, Elk Grove Village, USA; ${ }^{2}$ American Academy of Dermatology, Schaumburg, USA; ${ }^{3}$ Society of Critical Care Medicine, Mt Prospect, USA

\section{0:1136/bmjqs-2013-002293.228}

Background The Council of Medical Specialty Societies (CMSS) approved "Principles for the Development of Specialty Society Clinical Guidelines" as a set of standards that member societies could draw upon in developing their own development methodologies. Developed by the member societies in late 2012, the Principles are intended to provide a degree of interpretive flexibility not offered by other standard sets but based upon an expected level of transparency that individual interpretations would be explained.

Objectives Principles were labelled as "must", "should" and "may" in an effort to impart suggested implementation flexibility as designed by the Principles development team. This proposed analysis of society feedback is intended to assess the actual concordance with the Principles by societies implementing their methodologies or updating them.

Methods An electronic survey was sent to Societies who identified themselves as creating new or adapting existing methodologies asking agreement on ease of implementation in 10 previously identified contentious standards. The survey will also include opportunity for responders to identify other difficult to implement/interpret principles as well as new principles that should be considered.

Results Data from respondents will be presented.

Discussion Medical Specialty Societies developing clinical practice guidelines are diverse in terms of size, scope and resources. 